\title{
1 LAI, fAPAR and fCover CYCLOPES global products derived
}

\section{2 from VEGETATION. Part 1: Principles of the algorithm}

3
${ }^{1}$ INRA-CSE, Agroparc, 84914 Avignon, France

${ }^{2}$ CNRM, Toulouse, France

${ }^{3} \mathrm{CNES}$, Toulouse, France

${ }^{4}$ Medias-France, Toulouse, France

${ }^{5}$ Noveltis, Toulouse, France

\section{Abstract}

This article describes the algorithmic principles used to generate $L A I, f A P A R$ and fCover estimates from VEGETATION observations. These biophysical variables are produced globally at 10 days temporal sampling interval under lat-lon projection at $1 / 112^{\circ}$ spatial resolution. After a brief description of the VEGETATION sensors, radiometric calibration process, based on vicarious desertic targets is first presented. The cloud screening algorithm was then fine tuned using a global network of cloudiness observations. Atmospheric correction is then achieved using the SMAC code with inputs coming from meteorological values of pressure, ozone and water vapour. Aerosol optical thickness is derived from MODIS climatology assuming continental aerosol type. The Roujean BRDF model is then adjusted for red, near infrared and short wave infrared bands used to the remaining cloud free observations collected over a time window of \pm 15 days. Outliers due to possible cloud contamination or residual atmospheric correction are iteratively eliminated and prior information is used to get more robust estimates of the three BRDF kernel coefficients. Nadir viewing top of canopy reflectance in the three bands is input to the biophysical algorithm to compute the products at 10 days sampling interval. This algorithm is based on training neural networks over SAIL+PROPSPECT radiative transfer model simulations for each biophysical variable. 
1 Details on the way the training data base was generated and the neural network designed and 2 calibrated are presented. Finally, theoretical performances are discussed. Validation over 3 ground measurement data sets and inter-comparison with other similar biophysical products 4 are presented and discussed in a companion paper. The CYCLOPES products and associated 5 detailed documentation are available at http://postel.mediasfrance.org.

6 Key words: LAI, fAPAR, fCover, 


\section{$1 \quad 1$ Introduction}

2 Many applications require an exhaustive monitoring of surface characteristics including

3 biogeochemical cycle and climate modelling, resource evaluation (water, agriculture or forest production). Surface process models need a large number of inputs and parameters that depend on plant functional types. These relatively complex models need to be validated, calibrated or 'corrected' through assimilation of a number of measured variables. Among those, canopy structure characteristics, LAI (leaf area index) and fAPAR (fraction of photo-synthetically active radiation absorbed by the canopy) are key variables that are both used in surface process models and retrieved from remote sensing observations in the reflective solar domain. Other applications such as change detection in land cover due either to hazards, climatic variation or changes in land use (deforestation, aforestation, reforestation, changes in species or cultural practices), require a relatively high frequency monitoring of the Earth surface. Variables such as the previous ones in addition to fCover (vegetation cover fraction) retrieved from remote sensing observations, may offer a promising alternative to vegetation indices that are currently used although they did not relate simply to canopy biophysical variables.

For almost 25 years, the Earth surface has been monitored at medium to coarse resolution (not better than $1 \mathrm{~km}^{2}$ pixels) thanks to the series of NOAA/AVHRR sensors. More recently, new sensors have been launched with improved spectral (e.g. SEAWIFS/VEGETATION/MODIS/MERIS) or directional (e.g. POLDER/MISR) sampling, and higher spatial resolution (e.g. MERIS/MODIS). However, space agencies were used to provide only low level products such as top of atmosphere reflectances, sometimes top of canopy reflectances and vegetation indices, but no true biophysical variables such as $L A I$, AAPAR or fCover. Biophysical products derived from the observations of a selection of these sensors have just been recently developed, and made available to the scientific community. Modellers may now download these products from service centres and plug them directly into their applications. This is the basis of the success of MODIS products.

The Global Monitoring of the Environment and Security programme was initiated few years ago by Europe to better coordinate the use of satellite Earth observations. This programme is now considered as the European component of GEOSS (Global Earth Observation System of Systems) as defined GEO (Group of Earth Observation). Within GMES, Europe supported projects aiming at the development of biophysical products from currently available sensors. The CYCLOPES project was aiming at the development and actual production of global fields of $L A I$, fAPAR and fCover for the 1998-2003 period. These products were evaluated by associated users for applications focusing on carbon cycle, climate modelling and change detection. 
2 Although the CYCLOPES project was ultimately targeting to develop products from the fusion of an ensemble of satellites, one of the first tasks achieved was to develop specific products for each series of sensors. This paper present the results obtained for the VEGETATION sensor. After briefly presenting the characteristics of VEGETATION observations, and the way it is radiometrically calibrated, several steps required to derive the biophysical products from the low level products available at VITO ( http://www.vgt.vito.be ) will be described. It includes cloud screening, atmospheric correction, BRDF normalization, temporal compositing, and the derivation of the biophysical variables and some theoretical validation elements. A companion paper will present the actual validation of these products with some inter-comparison with MODIS Collection 4 (Weiss et al., 2007).

\section{VEGETATION sensors characteristics and radiometric} calibration

\subsection{VEGETATION sensors characteristics}

Europe has launched the first VEGETATION sensor in 1998 aboard SPOT4. A second instrument was launched in 2002 aboard SPOT5 to ensure the continuity of observations. The two instruments are identical and provide global observations of the surface from a sun-synchronous orbit at $822 \mathrm{~km}$ altitude, with an inclination of $96.7^{\circ}$, a period of 26 days and an equatorial crossing time of 10:30. Because of the large swath ( $101^{\circ}$, equivalent to $2200 \mathrm{~km}$ ), about $90 \%$ of the equatorial areas are imaged each day, the remaining $10 \%$ being imaged the next day. For latitudes higher than $35^{\circ}$ (North and South), all regions are acquired at least once a day. The instrumental concept relies on a linear array of 1728 CCD detectors providing a spatial resolution around $1.15 \mathrm{~km}$ with minimum variations for off-nadir pixel size thanks to the telecentric design of the optics. Four spectral bands are available: B0 (450 nm, $\Delta \lambda=40 \mathrm{~nm}) ;$ B2 (645 nm, $\Delta \lambda=70 \mathrm{~nm})$; B3 (835 nm, $\Delta \lambda=110 \mathrm{~nm})$; SWIR (1165 nm, $\Delta \lambda=170 \mathrm{~nm}$ ). The stability of the platform, the accurate knowledge on its position and attitude and post processing of the images allow to achieve a multi-temporal registration accuracy around $200 \mathrm{~m}$ (rms). (Sylvander et al., 2003). The system and the corresponding products are described with more details in (Henry, 1999) and (Maisongrande et al., 2004). The P products kindly copied from the archive stored at the VITO processing and archiving centre in Mol (Belgium) were used within this CYCLOPES project. $P$ products are extracts of a segment along a single orbit. $P$ products are geometrically and radiometrically corrected and correspond thus to top of atmosphere reflectance. These products are delivered under the lat-lon projection at $1 / 112^{\circ}$ spatial 


\section{$1 \quad 2.2$ Radiometric calibration}

2 CYCLOPES project was aimed at processing data from many sensors with consistent algorithms. The

3 first step consists in obtaining a common calibration reference, thus giving priority to a cross

4 calibration of sensors compared to an absolute calibration. Calibration over desert sites is well suited

5 for this purpose because it provides large extent homogeneous areas to minimise misregistration, a

6 temporal stability, low variability of the overlying atmosphere including low cloudiness, and reduced

7 directional effects. Twenty areas, each larger than $100 \times 100 \mathrm{~km}^{2}$ in North Africa and Saudi Arabia

8 corresponding to these criteria were selected (Cosnefroy et al., 1996). The POLDER instruments

9 offering a very good characterisation of the site Bi-Directional Reflectance Factor (BRDF) in 8

10 spectral bands (443nm to $910 \mathrm{~nm}$ ) were used as the reference sensors. Each site observed by 11 VEGETATION may be also observed by POLDER under very similar directional configuration.

12 POLDER in-flight calibration has been thoroughly studied (Hagolle et al., 1999) and its accuracy is 13 estimated better than 5\% (3 sigma). POLDER 2 instrument was thus used here to cross-calibrate other 14 sensors including VEGETATION. More details on the desert cross calibration method can be found in 15 (Cabot et al., 2000; Hagolle et al., 1999).. However, for VEGETATION SWIR band, it is not possible 16 to use POLDER as a reference since it has no spectral band in the SWIR domain. In this case, the reference sensor is VEGETATION 1 (Hagolle et al., 2004). Continuous monitoring of these desertic sites allows updating the original radiometric calibration coefficients by adjusting an empirical model accounting for the degradation of performances of the sensor (Figure 1). Note that a non linear model

20 based on the sum of an exponential model describing the more rapid evolution of the optical system 21 just after launch particularly important in the blue band, and a linear model describing the slow 22 degradation of the system. 

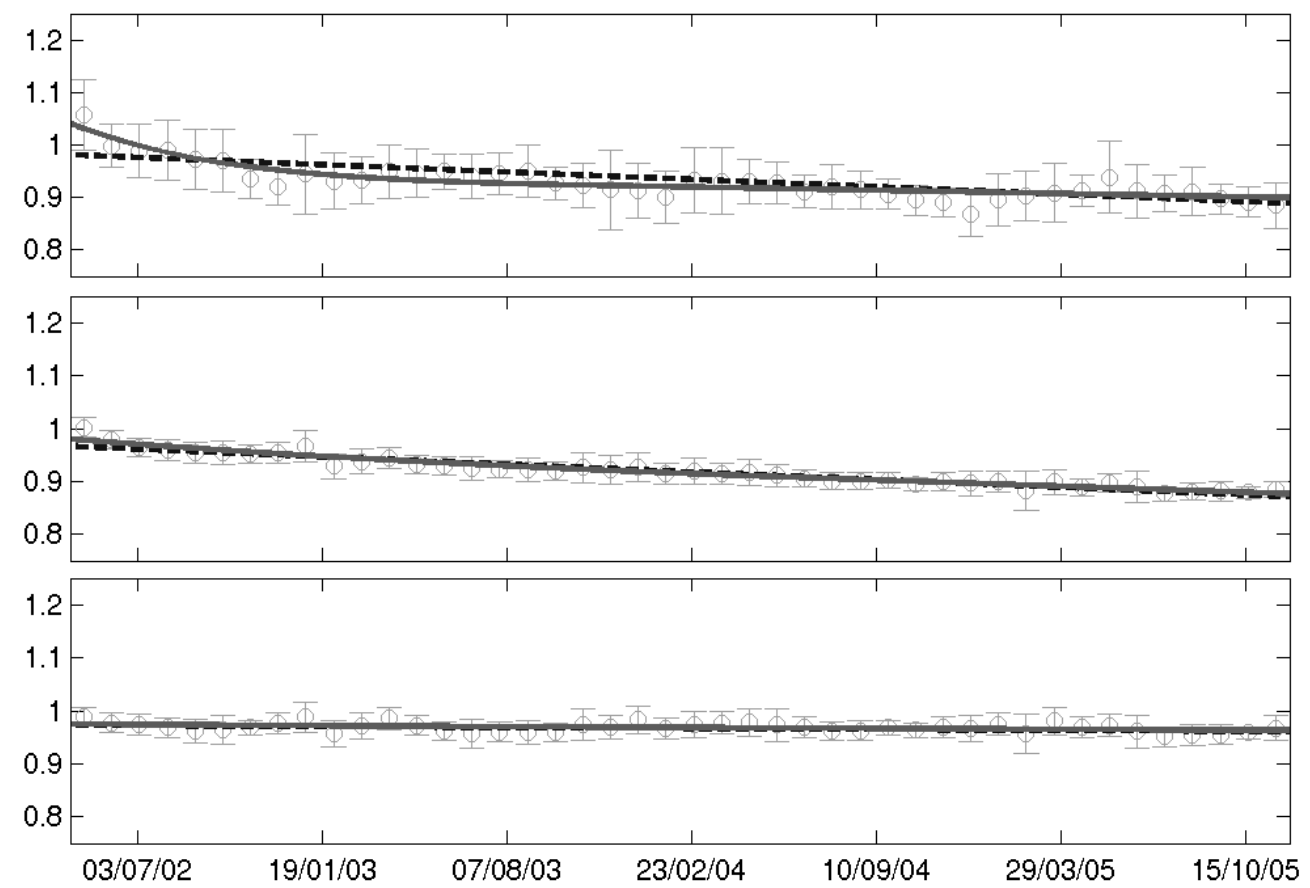

4 Figure 1. Time course of the calibration coefficient obtained using 20 desert sites (Cabot et al., 2000; Hagolle et al., 1999) for VEGETATION 2 using POLDER 2 as a reference: top: Blue band (B0); centre: red band (B2); bottom: near-infrared band (B3). The calibration coefficient corresponds here to the correction to apply to the pre-launch calibration of VEGETATION2. Symbols are monthly averages with standard deviation, and curves are the best fit of a linear model (dashed line) or the non linear model (plain line).

\section{Principles of the algorithm}

Top of atmosphere calibrated reflectance values were transformed into top of canopy reflectance values computed in a standard geometrical configuration and for evenly spaced dates. These top of canopy directionally normalized reflectance values are used as inputs to the biophysical algorithm to get LAI, fAPAR and fCover estimates. Four steps are therefore necessary to get the biophysical products. They include chronologically cloud screening, atmospheric correction, BRDF normalization and temporal compositing and finally biophysical algorithm. These four steps are described hereafter.

\subsection{Cloud screening}

17 Cloud screening for VEGETATION as defined by Kempeneers et al. (2000) uses a constant threshold on blue band (B0) based on Top Of Atmosphere reflectance $\left(\mathrm{R}^{\mathrm{TOC}}(\mathrm{B} 0)\right)$. In this domain, clouds are relatively bright because of the importance of scattering, while surfaces are generally dark because of strong absorption features of many vegetation and soil constituents. Such threshold provides good results globally, regardless of both surface type and viewing geometry. However, performances are limited over bright surfaces. Hagolle et al. (2005) developed an alternative cloud screening method based on surface reflectance computed using the SMAC code (Rahman and Dedieu, 1994) and 
corrected only from Rayleigh scattering and gaseous absorption. This allows accounting for first order effects in the observation geometry. Because threshold values in B0 may depend on land cover, the 1 $\mathrm{km}$ spatial resolution GLC2000 global land cover classification was used to provide this piece of information (Bartholomé and Belward, 2005). Specific thresholds were adjusted empirically for barren, sparse herbaceous, artificial surfaces and associated areas, mosaic cropland/shrub cover, and shrub cover classes were tuned: the pixel is flagged as cloudy if $\left(\mathrm{R}^{\mathrm{TOC}}(\mathrm{B} 0)>0.24\right)$ and $\left(\mathrm{NDVI}^{\mathrm{TOC}}<0.05\right)$. For the other classes, the algorithm of Hagolle et al. (2005) is applied. To separate snow from clouds a snow detection algorithm was finally implemented based on spectral variation of snow (Dozier, 1989). Thresholds associated to each of the four bands were empirically tuned (Berthelot, 2004). Note that observations partly saturated in B0 and B2 are taken into account for snow detection. Note also that cloud detection is not active over salt lakes.

Cloud screening was validated at global scale during April 1999 by comparison to quasi-simultaneous cloud fraction observations observed over 11500 synoptic nebulosity ground stations provided by Météo-France (Berthelot et al., 2004). Comparison with CYCLOPES cloud mask shows good performances over Europe, with however some over detection above snow, and under detection in tropical regions, due to the difficulty to identify semi transparent clouds.

Once cloudy pixels are detected, cloud shadows are first projected over the surface taking into account view and sun directions, assuming that clouds are at a $5 \mathrm{~km}$ altitude. The cloud mask is spatially extended over a 2 x 2 pixels radius to prevent small contamination of clear pixels in the cloud vicinity. Pixels are then declared 'suspect' over a 3 x 3 radius centred on the cloud detected pixels. Note that cloudy pixels are not spatially extended over snowy pixels. Each valid pixel (not cloudy, not shadowed, not saturated or not out of orbit) may thus be declared either clear (all the bands valid), suspect (in the vicinity of cloudy/shadowed pixels), or snowy (with all the bands valid or with just B0 and/or B2 bands invalid).

\subsection{Atmospheric correction}

Atmospheric effects are corrected using the SMAC code (Rahman and Dedieu, 1994) for the four VEGETATION bands. Input atmospheric characteristics are derived from currently available products: NCEP Meteorological data for pressure and water vapour, and TOMS/TOVS for ozone (ftp://oceans.gsfc.nasa.gov). With the availability of MODIS aerosol products (Kaufman et al., 1997), the possibility to achieve better atmospheric correction based on monthly mean values (MOD08_M3) was investigated. The climatology was built over the period covering March 2000 up to August 2003, representing 42 months. Performances of the corresponding monthly $1^{\circ} \times 1^{\circ}$ spatial resolution MODIS 
over an ensemble of 250 sites distributed globally. The monthly average of aerosol optical thickness at $550 \mathrm{~nm}\left(\mathrm{AOT}_{550}\right)$ was computed from Level 2.0 AERONET products.

Results show significant overestimation of MODIS monthly $1^{\circ} \times 1^{\circ}$ spatial average as compared to cloud free AERONET measurements averaged over the month (Figure 2, left). This confirms previous observations achieved over daily aerosol products (Béal et al., 2006; Levy et al., 2005). Inspection of the yearly dynamics of mean, maximum and minimum MODIS monthly $\mathrm{AOT}_{550}$ values shows that the minimum of the monthly values was in closer agreement with AERONET monthly AOT $_{550}$ time course. Figure 2 (right) shows that while significant scattering is observed between MODIS and AERONET values, no systematic biases is observed. Sensitivity analysis of atmospheric correction performances achieved by comparing VEGETATION 1 and 2 top of canopy reflectance Hagolle et al. (2005) showed improvements as compared to the mean monthly values or to the simple latitudinal gradient proposed by Berthelot and Dedieu (1997). The minimum of the monthly values was thus proposed to be used for atmospheric correction. However, MODIS aerosol products present missing values and unexpected large $\mathrm{AOT}_{550}$ values $\left(\mathrm{AOT}_{550}>0.6\right)$ that were removed, mostly corresponding to transition between deserts and vegetated areas. The gaps were thus filled with the simple latitudinal gradient proposed by Berthelot and Dedieu (1997). A spatial smoothing procedure is finally applied to minimize the possible discontinuities between $1^{\circ} \times 1^{\circ}$ pixels and to reach the original VEGETATION spatial resolution.
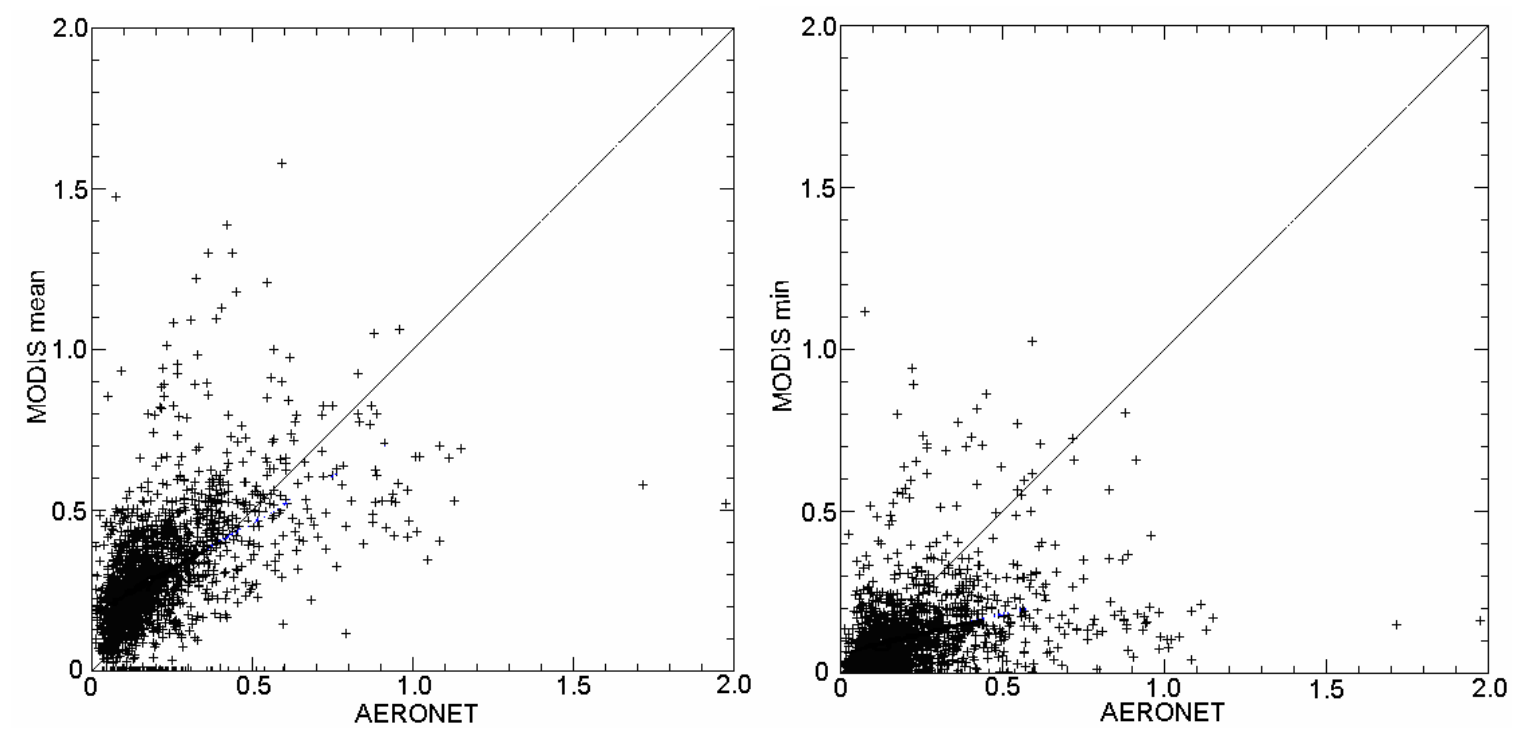

Figure 2. Comparison between MODIS monthly mean (left) and minimum (right) values averaged over $1^{\circ} \times 1^{\circ}$ areas and the monthly mean values of aerosol optical thickness at $550 \mathrm{~nm}$ measured from AERONET sun photometers for clear sky conditions.

\subsection{BRDF normalization and temporal compositing}

25 The objective of the directional normalization is to transform a set of reflectance factor measurements 26 taken at irregularly spaced dates under varying view and solar angles into a regular time series of 
1 parameters describing the BRDF. The principles behind this algorithm have been already presented in

2 Hagolle et al. (2005). However, additional improvements have been implemented including a better

3 weighing of observations, prior constraints on LAI and the reduction in the minimum number of

4 required observations as well as accounting for snow cover. These improvements of the original

5 algorithm are presented hereafter.

\subsubsection{Reflectance Model}

Roujean’s model linear BRDF model (Roujean et al., 1992) was used for processing top of canopy reflectance data $R\left(\theta_{v}, \theta_{s}, \phi\right):$ It is a where reflectance depends on:

$$
R\left(\theta_{v}, \theta_{s}, \phi\right)=k_{0}+k_{1} f_{1}\left(\theta_{v}, \theta_{s}, \phi\right)+k_{2} f_{2}\left(\theta_{v}, \theta_{s}, \phi\right)
$$

$\theta_{v}$ and $\theta_{s}$ are the view and sun zenith angles and $\phi$ the relative azimuth angle between the two directions and $k_{i}$ are coeffcients to be tuned for each band. Functions $f_{1}$ and $f_{2}$ are fixed angular kernels representing the angular distribution related to "geometric" and "volumetric" surface scattering processes. The technique used to estimate the $k_{i}$ coefficients is described in detail in Geiger et al. (Geiger et al., 2006) and is based on a customized linear regression. The main principles are summarized below.

\subsubsection{Weighting reflectance observations}

A weight factor $w$ depending on time, view and sun directions is associated to each reflectance observation collected in the temporal compositing window, and used in the linear regression:

$$
w=w_{\theta}\left(\theta_{v}, \theta_{s}\right) \cdot w_{t}(t)(2)
$$

The angular component of the weight, $w_{\theta}\left(\theta_{v}, \theta_{s}\right)$ is assumed to be inversely proportional to the corresponding uncertainties $\sigma_{R}$. Atmospheric correction uncertainties contribute the more to the noncorrelated (random) part of the TOC-reflectance errors and was therefore set proportional to the airmass $\eta\left(\theta_{v j}, \theta_{s j}\right)$ and uncertainties for nadir viewing and illumination geometry, $\sigma_{R(0,0,0)}$.

$$
w_{\theta}\left(\theta_{v}, \theta_{s}\right)=\frac{1}{\sigma_{R}}=\frac{1}{\eta\left(\theta_{v}, \theta_{s}\right) \sigma_{R(0,0,0)}}=\frac{2}{\left(\frac{1}{\cos \theta_{v}}+\frac{1}{\cos \theta_{s}}\right) \sigma_{R(0,0,0)}}
$$

As described in (Geiger et al., 2006), the value of $\sigma_{R(0,0,0)}$ can be approximated as a linear function of reflectance $R$ : $\sigma_{R(0,0,0)}=c_{1}+c_{2} R$ with coefficients $c_{1}$ and $c_{2}$ given in Table 1 . 


\begin{tabular}{|l|l|l|}
\hline Bands & $C_{1}$ & $C_{2}$ \\
\hline Blue & 0.009 & 0.14 \\
\hline Red & 0.005 & 0.05 \\
\hline NIR & 0.003 & 0.03 \\
\hline SWIR & 0.005 & 0.03 \\
\hline
\end{tabular}

1 Table 1. Values of $c_{1}$ and $c_{2}$ coefficients to approximate the error $\sigma_{R(0,0,0)}$ for the four VEGETATION bands.

2 The temporal component of the weight $w_{t}(t)$ was designed to put more emphasis on data collected 3 close to the centre $t_{0}$ of the temporal window of width $\tau$ (in days) where all observations are collected 4 to adjust the BRDF model. A gaussian function centred on $t_{0}$ and truncated at $\pm \tau / 2$ is used to compute $w_{t}(t)$. The standard deviation of the gaussian was selected so that $w_{t}(t \pm \tau / 2)=0.5$ to provide enough weight at the extremities of the window. A compromise was found between the resulting number of cloud-free observations typically available and the user requirements on the temporal resolution of the products. A value of $\tau=30$ days was chosen, corresponding thus to the temporal resolution. Note that pixels flagged as 'suspect' are used in the compositing period.

\subsubsection{Contraints on $k_{i}$ coefficients}

Prior values of $k_{1}$ and $k_{2}$ coefficients were computed as the average of the estimated values derived by adjusting the BRDF model without constraints over a representative set of VEGETATION data. These values (Table 2) were then introduced as a priori constraints in the model inversion process. Taking into account this prior information of the form $k_{i}=k_{i \text { ap }} \pm \sigma_{\text {ap }}\left[k_{i}\right]$ considerably reduces the uncertainties in $k_{i}$ estimates when the directional sampling achieved with the available observations is limited. It also increases the efficiency of the outlier elimination scheme described below. Note that a minimum of two valid observations in the compositing window was required.

\begin{tabular}{|l|l|l|l|l|}
\hline Bands & $k_{1 \text { ap }}$ & $\sigma_{\text {ap }}\left[k_{1}\right]$ & $k_{\text {2 ap }}$ & $\sigma_{\text {ap }}\left[k_{2}\right]$ \\
\hline Blue (B0) & 0.00 & 0.07 & 0.08 & 0.29 \\
\hline Red (B2) & 0.02 & 0.05 & 0.17 & 0.30 \\
\hline NIR (B3) & 0.04 & 0.07 & 0.67 & 0.34 \\
\hline SWIR & 0.05 & 0.06 & 0.41 & 0.28 \\
\hline
\end{tabular}

Table 2: A priori information on $k_{1}$ and $k_{2}$ coefficients of Roujean's BRDF model for the four bands of VEGETATION instrument bands.

\subsubsection{Iterative elimination of outliers}

Undetected clouds can introduce non-Gaussian outliers in the error structure of reflectance and decrease the performances of the inversion process. An iterative scheme for eliminating unreliable observations as described in Hagolle et al. (2005) was therefore implemented. Detection of outliers is 
1 residual clouds. Those outlier dates are then eliminated in all bands. The decision criterion for

2 elimination is based on the "relative error", $\sigma_{\text {rel }}$ defined as:

$$
\sigma_{\text {rel }}=\sqrt{\frac{\sum_{t=1}^{n}(\hat{R}(t)-R(t))^{2}}{\sum_{t=1}^{n}(\bar{R}-R(t))^{2}}}
$$

4 Where $\hat{R}(t)$ is the estimated reflectance for observation date $t$, and $\bar{R}$ is the reflectance value averaged over the $n$ available observations considered within the temporal window. If $\sigma_{\text {rel }}$ computed after the first iteration exceeds the threshold value $\sigma_{t h r}=0.25$ then all observations exceeding the model fit by more than $\sqrt{\frac{1}{n} \sum_{t=1}^{n}(\hat{R}(t)-R(t))^{2}}(1$ standard deviation) are eliminated.

Otherwise and if $\sigma_{r e l}>0.5 \sigma_{t h r}$, all observations deviating from the model fit by more than $2 \sqrt{\frac{1}{n} \sum_{t=1}^{n}(\hat{R}(t)-R(t))^{2}}$ (2 standard deviation) are eliminated. The model fit is then repeated and the relative error $\sigma_{r e l}$ is recalculated. The procedure is iterated up to four times as long as $\sigma_{r e l}>0.5 \sigma_{t h r}$ and the number of eliminated observations does not exceed one third of the initially available ones. Note that temporal and angular weighing is applied only for the last iteration to save computation time. Note that the threshold values were empirically derived and additional tests have shown that the results were not too sensitive to their precise values which explains the simple numbers used $(0.25$ and 0.5$)$.

\subsubsection{Snow pixels}

Reflectance properties of land surfaces can be drastically changed over small time scales by snow fall and melt. To prevent instabilities in the inversion due to reflectances with inconsistent values, it has to be decided whether the window is considered mainly with or without snow. If less than half of the total number of reflectance observations during a reduced period of ten days centred on $t_{0}$ is flagged as snow-covered, then all observations over the full compositing period flagged as snowy are eliminated and the inversion is performed only with the snow-free observations. Conversely, when the majority of observations are flagged as 'snow', the snow-free reflectances are eliminated and the BRDF model adjustment is applied only over snow observations. If the number of snow-free equals that of snow-covered observations, the full composition period is used for the decision criterion.

\subsection{Biophysical algorithms}

The last three steps allow getting relatively clean top of canopy reflectance values in a standard geometrical configuration (nadir viewing, sun zenith angle corresponding to the median value in the 
estimate the corresponding $L A I$, fAPAR and fCover biophysical variables. Before describing the biophysical algorithms, a clear definition of CYCLOPES biophysical products must be given:

- LAI: the CYCLOPES products correspond to some effective $L A I$ where only clumping at the landscape level is accounted for: pixels are considered made of patches of different surface types. This alteration of the main definition (half the developed area of green vegetation elements per unit horizontal soil) is due to the radiative transfer models and the embedded assumptions on canopy architecture considered as explained later.

- fAPAR: The fAPAR CYCLOPES products correspond to a black-sky value (no diffuse illumination) at 10:00 local solar time. It is a fair approximation of the daily integrated value for non cloudy days (Baret et al., 2004).

- fCover refers only to the green vegetation elements.

The biophysical algorithm is based on radiative transfer model inversion. A neural network approach was selected for many reasons: neural networks are known to be computationally very efficient, which is very important for operational applications over a long time series of global data. A recent review on canopy characteristics retrieval methods (Baret and Buis, 2007) showed that neural networks when trained over radiative transfer model simulations provides good estimation performances because of their efficient interpolation capacity (Leshno et al., 1993). This model inversion technique has been applied with success to remote sensing of land surfaces since about a decade (Abuelgasim et al., 1998; Atkinson and Tatnall, 1997; Baret et al., 1995; Danson et al., 2003; Fang and Liang, 2005; Gong et al., 1999; Kimes et al., 1998; Smith, 1992, 1993; Weiss et al., 2002). Recently, several authors developed operational biophysical products for medium resolution sensors, with top of canopy reflectance as inputs: (Lacaze, 2005) for POLDER, (Bacour et al., 2006) for MERIS.

Algorithm calibration consists in generating a learning database from which the network architecture and the synaptic coefficients and bias are tuned. The radiative transfer model used, distribution of the input variables will first be described. Calibration of the network as well as management of extreme cases will then be presented. Note that our algorithm is designed to be applied to any surface type conversely to what is implemented for MODIS (Myneni et al., 2002) or GLOBCARBON (Plummer et al., 2005) products for which the algorithm is tuned for each biome type.

\subsubsection{Generating the learning data base}

Radiative transfer models were run to simulate the actual VEGETATION observations. The SAIL model (Verhoef, 1984, 1985) with the hot-spot correction implemented by (Kuusk, 1991) was used. It assumes the canopy as a turbid medium for which leaves are randomly distributed in space. Canopy structure is characterized by $L A I$, the average leaf angle inclination (ALA) assuming an ellipsoidal distribution (Campbell, 1990) and the hot-spot parameter. To account for clumping at the landscape scale, each pixel was supposed to be made of a fraction vCover of pure vegetation and (1-vCover) of 
pure bare soil. The extensive biophysical variables (LAI, fAPAR, fCover) and reflectances were thus computed at the pixel level according to this assumption.

Leaf optical properties are simulated with the PROSPECT model (Jacquemoud and Baret, 1990) using specific absorption coefficients calibrated by (Fourty and Baret, 1997). It requires 5 input variables: the mesophyll structure parameter $(N)$, chlorophyll $(C a b)$, dry matter $(C d m)$, water $(C w)$ and brown pigment $(\mathrm{Cbp})$ contents. Water content was tied to the dry matter content assuming that green leaves have a relative water content $(H)$ varying within a relatively small range (Table 3 ).

Reflectance values of soils were simulated using 5 typical soil reflectance spectra multiplied by a brightness coefficient $B_{s}$. These 5 reflectance spectra (Figure 3) were selected to represent with a good accuracy within VEGETATION bands (RMSE $<0.01)$ the reflectance spectra of a large soil database where soil types, roughness, moisture and observational geometry vary over a large range (Liu et al., 2003). The distribution of the brightness coefficient observed over this data base was used in the generation of the training database. Note that here again selection of the soil type and brightness coefficient is independent on any prior knowledge of the surface type.

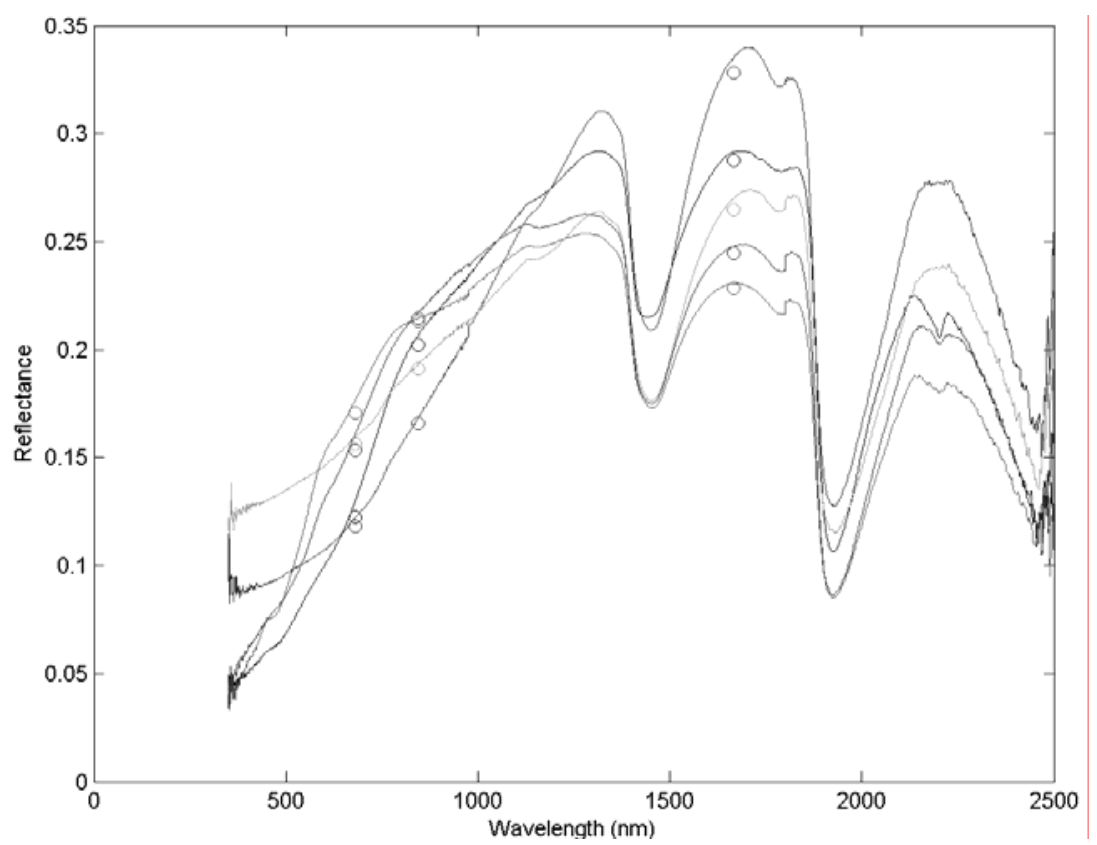

Figure 3. Reflectance of the 5 soils used to represent the possible range of spectral shapes.

Table 3 shows the distribution of all the input variables. Note that for LAI, a uniform distribution law was used in order to put more emphasis on the larger LAI values that are more difficult to retrieve. Truncated gaussian or uniform distributions were used for the other variables, trying to describe the actual distribution over the Earth. Observation geometry was derived from computing orbit dynamics of VEGETATION assuming uniform distribution of sites in latitude and longitude as well as date of 
1 observations (Table 3). A full orthogonal experimental plan was used to combine the 13 input

2 variables by splitting the whole range of variation of each variable into a small number of classes. This

3 resulted to 196608 cases simulated.

\begin{tabular}{|c|c|c|c|c|c|c|c|c|}
\hline & Input variables & Unit & $\min$ & mean & $\max$ & std & law & Class \\
\hline \multirow{3}{*}{$\begin{array}{l}\text { Observation } \\
\text { geometry }\end{array}$} & Latitude & $\circ$ & $-60^{\circ}$ & $0^{\circ}$ & $60^{\circ}$ & - & Uniform & 1 \\
\hline & Longitude & $\circ$ & $0^{\circ}$ & $180^{\circ}$ & $360^{\circ}$ & - & Uniform & 1 \\
\hline & Day of year & day & 1 & 92 & 183 & - & Uniform & 1 \\
\hline \multirow{4}{*}{$\begin{array}{l}\text { Canopy } \\
\text { structure }\end{array}$} & LAI & - & 0 & 3 & 6 & - & Uniform & 6 \\
\hline & ALA & $\circ$ & $30^{\circ}$ & $60^{\circ}$ & $80^{\circ}$ & $20^{\circ}$ & Gauss & 4 \\
\hline & Hot & - & 0.001 & 0.1 & 1 & 0.3 & Gauss & 1 \\
\hline & vCover & - & 0 & 1 & 1 & 0.2 & Gauss & 2 \\
\hline \multirow{5}{*}{$\begin{array}{l}\text { Leaf optical } \\
\text { properties }\end{array}$} & $\mathrm{N}$ & - & 1 & 1.5 & 2.5 & 1 & Gauss & 4 \\
\hline & $\mathrm{Cab}$ & $\mu \mathrm{g} \cdot \mathrm{cm}^{-2}$ & 30 & 50 & 90 & 30 & Gauss & 4 \\
\hline & $\mathrm{Cdm}$ & g.cm ${ }^{-2}$ & 0.002 & 0.0075 & 0.02 & 0.0075 & Gauss & 4 \\
\hline & $\mathrm{H}$ & - & 0.65 & 0.75 & 0.85 & - & Uniform & 4 \\
\hline & Cbp & - & 0 & 0 & 1.5 & 0.2 & Gauss & 4 \\
\hline Background & Bs & - & 0.2 & 1 & 2.2 & 0.7 & Gauss & 4 \\
\hline
\end{tabular}

4 Table 3. Distribution of the input variables of the radiative transfer models used to generate the training database 5

6 For any combination of the input variables, top of canopy reflectance was computed for each wavelength and then integrated according to the spectral sensitivity of VEGETATION bands. The

8 blue band (B0) was not used here because it was considered too much contaminated by residual atmospheric effects. A white gaussian noise of 0.04 (absolute value) was added to the simulated reflectances to account for uncertainties attached to measurements and models. It is quite difficult to quantify precisely the uncertainties attached to top of canopy reflectances used as input to the biophysical algorithm since they are induced by several possible processes including radiometric 13 calibration, BRDF normalization, atmospheric correction, residual cloud contamination and of course the suitability of the radiative transfer models to describe photon transport in actual canopies. In addition uncertainties have some structure that is very difficult to evaluate that may however impact largely results. The uncertainties level selected for all the three bands corresponds to the order of magnitude of radiometric calibration (about 3 to $5 \%$ ), BRDF normalization (lower than 0.02 absolute), and 0.01 up to 0.10 for atmospheric corrections depending on assumptions and bands (Beal 2006). However, complementary sensitivity analysis was conducted showing a relatively small effect of the uncertainty level (in the range $0.00-0.05$ ) on theoretical performances. 

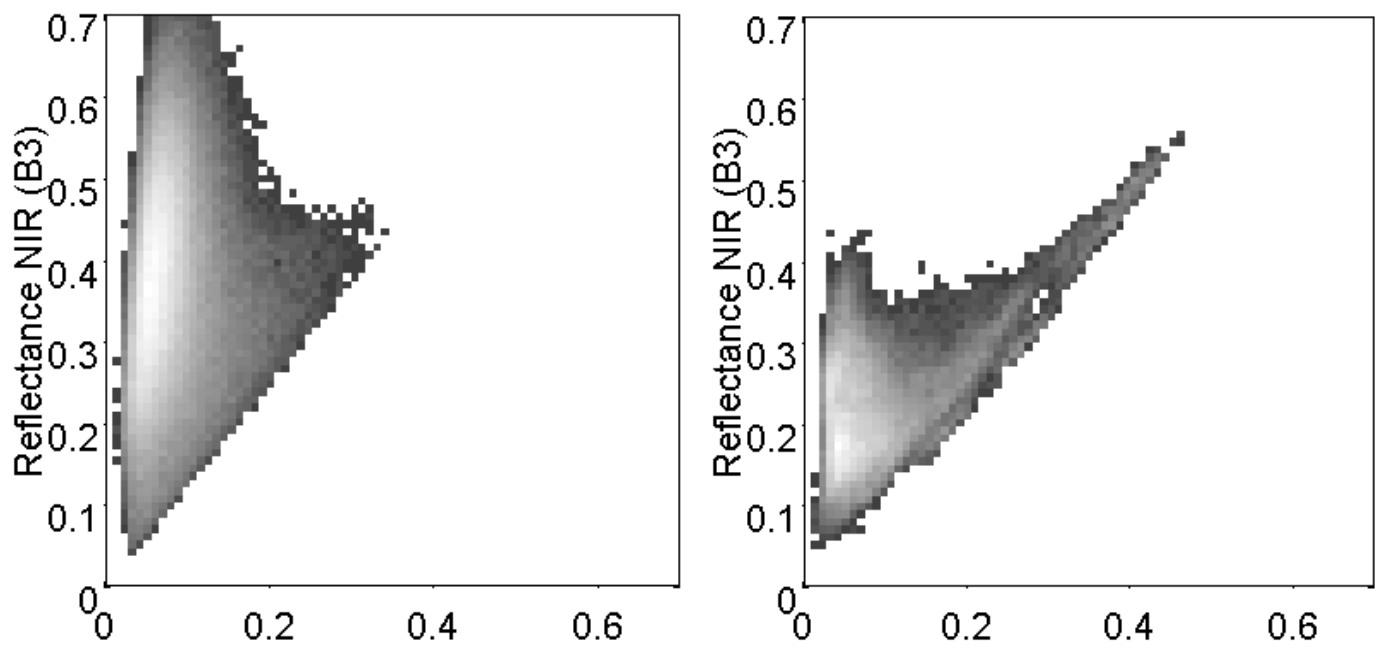

2 Figure 4. On the left, density plots of simulated reflectance values in red (B2) and near infrared (B3) feature

3 plan (196608 cases). On the right, the same figure with actual VEGETATION data acquired over a subset of

4196608 pixels randomly selected over the BELMANIP sites for years 1999-2003 and for which water bodies, cloud and snow contaminated pixels were eliminated as far as possible.

First evaluation of the simulations was achieved by simply plotting red and near infrared reflectance values. It shows (Figure 4, left) the typical pattern as expected, with the soil line bounding the bottom of the scatter plot. The realism of the simulated reflectance in the training database was further evaluated by comparison over a database of the same number of cases representing the possible range of variation of land surface properties: 196608 pixels were randomly selected in an ensemble of sites (BELMANIP, (Baret et al., 2006)) over the 1999-2003 period. Results show that the distribution of simulated cases (Figure 4, left) differs from that of actual VEGETATION observations (Figure 4, right): more cases are concentrated for low reflectance values in the red band, and higher near infrared reflectance values were simulated as compared to actual observations. These differences are due assumptions on the distribution and co-distribution of the variables in the training data base: for example, LAI distribution over the Earth is not uniform, and higher LAI values might not be observed with a planophile leaf inclination distribution (small ALA values), although these cases were simulated in a systematic way assuming the independency between LAI and ALA. Better agreement between simulated and actual distribution of reflectance data might improve the algorithm performances. Further efforts will have to be directed towards this issue.

However, more importantly is the realism with which reflectance data are simulated as compared with actual observations. The mismatch, $\delta$, between each VEGETATION measurements and the closest simulated reflectance was computed according to equation [5] over the ensemble $\Omega$ (196608 cases) of measured $\left(\rho_{b}\right)$ values for the three VEGETATION bands $(b)$ considered here. 


$$
\delta=\min _{\Omega}\left(\sqrt{\frac{\sum_{b=1}^{3}\left(\rho_{b}-\hat{\rho}_{b}\right)^{2}}{3}}\right)
$$

2 This was repeated for all the VEGETATION measurements collected previously (196608 pixels).

3 Figure 5 shows that most observations may be matched very closely with a simulated case. Note that 4 the assumed uncertainties level (0.04 RMSE value) is larger than the RMSE value associated to the 5 reflectance mismatch. This is mainly explained by the fact that model uncertainties are only 6 marginally described by the mismatch test that concentrates on reflectance values independently on the input canopy characteristics. Distribution of the residuals for each of the three bands confirms the consistency between simulated and observed reflectance values.

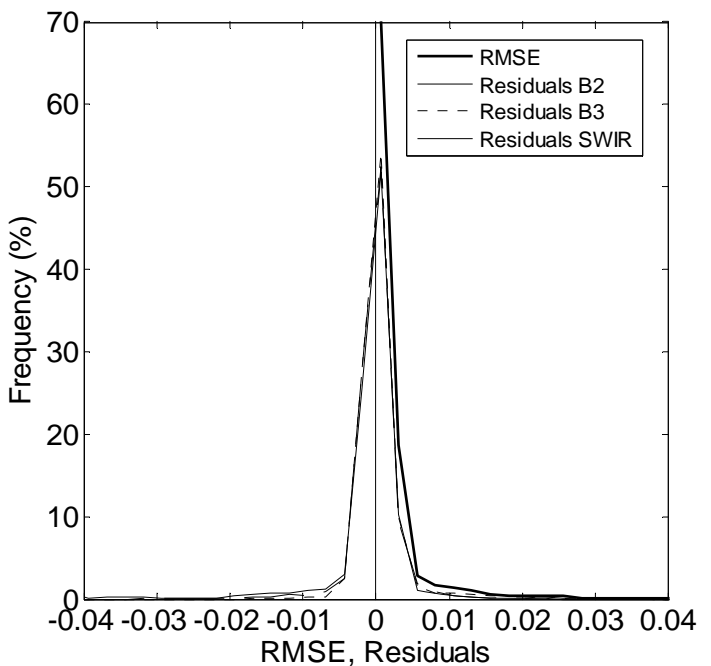

10 Figure 5. Distribution of the mismatch values $(\delta)$ as computed by equation [5] over a database made of 2000 VEGETATION observations.

Finally, fAPAR values for $\theta_{\mathrm{s}}$ at 10:00 solar time and fCover were also computed by running the radiative transfer code in the forward direction with the same input variables as those used for reflectance simulations.

\subsubsection{The networks} Inputs of the network are made of the median value of the sun zenith angle observed during the compositing period and top of canopy nadir reflectance in the three VEGETATION bands B2, B3 and SWIR. Outputs are the biophysical variables: LAI, fAPAR and fCover. The learning data base made of pairs of inputs and outputs was split in three parts by randomly selecting cases: half of the cases were used to train the network, a quarter to test the hyper-specialization during the training process. The last quarter was used for theoretical validation. Input and output $(X)$ data were first normalized 
1 ( $\left.X_{\text {Norm }}\right)$ according to: $X_{\text {Norm }}=2 *\left(X-X_{\text {Min }}\right) /\left(X_{\text {Max }}-X_{\text {Min }}\right)-1$ where $X_{\text {Max }}$ and $X_{\text {Min }}$ are respectively the

2 maximum and minimum values observed in the data base for variable $X$.

3

4

For each biophysical variable, a dedicated back-propagation artificial neural network (Rummelhart et al., 1986) was trained. After several tests, this solution was preferred to that consisting in a single network with three outputs as proposed by (Bacour et al., 2006). An optimal architecture was selected for each biophysical variable which converged towards the one depicted by Figure 6. For each variable, five networks were trained with different initial solutions, and the one providing the best performances (over the validation data set) was selected. The Levenberg-Marquardt minimization algorithm was used in the learning process because of its convergence performances (Ngia and Sjoberg, 2000). Note that 26 synaptic and 6 biases coefficients needed to be adjusted, which corresponded to about 3000 training data for one coefficient to tune which was far above the minimum value advised by (Harrel, 2001). 4

(5)

Figure 6. Architecture of the neural network used to estimate LAI, fAPAR and fCover (here labelled Var) from VEGETATION normalized top of canopy reflectance in bands B2, B3 and SWIR. Sun zenith angle $\left(\theta_{\mathrm{s}}\right)$ corresponding to the median value during the compositing period is also used as input. 'Norm' corresponds to the normalization of the inputs and outputs. Neurones with ' $S$ ' and ' $L$ ' mean respectively tangent-sigmoid and linear transfer functions.

\subsubsection{Theoretical performances}

The theoretical performances were evaluated over the validation data set made of 25\% (37850 cases)

3 of the simulated cases extracted from the database. It shows gradually improved performances (Figure 4 7) from $L A I$ (RMSE=1.10; RRMSE=37\%), fAPAR (RMSE=0.08; RRMSE=12\%) and $f$ Cover (RMSE=0.06; RRMSE=10\%). The poorest performances observed for $L A I$ are mainly due to reduced sensitivity of reflectances to $L A I$ for the larger $L A I$ values. Estimated $L A I$ values always keep lower 7 than $L A I=5.5$, which is probably due to the regularization of the inversion process achieved with the 28 distribution of $L A I$ values in the training data base as noticed by (Baret and Buis, 2007). 

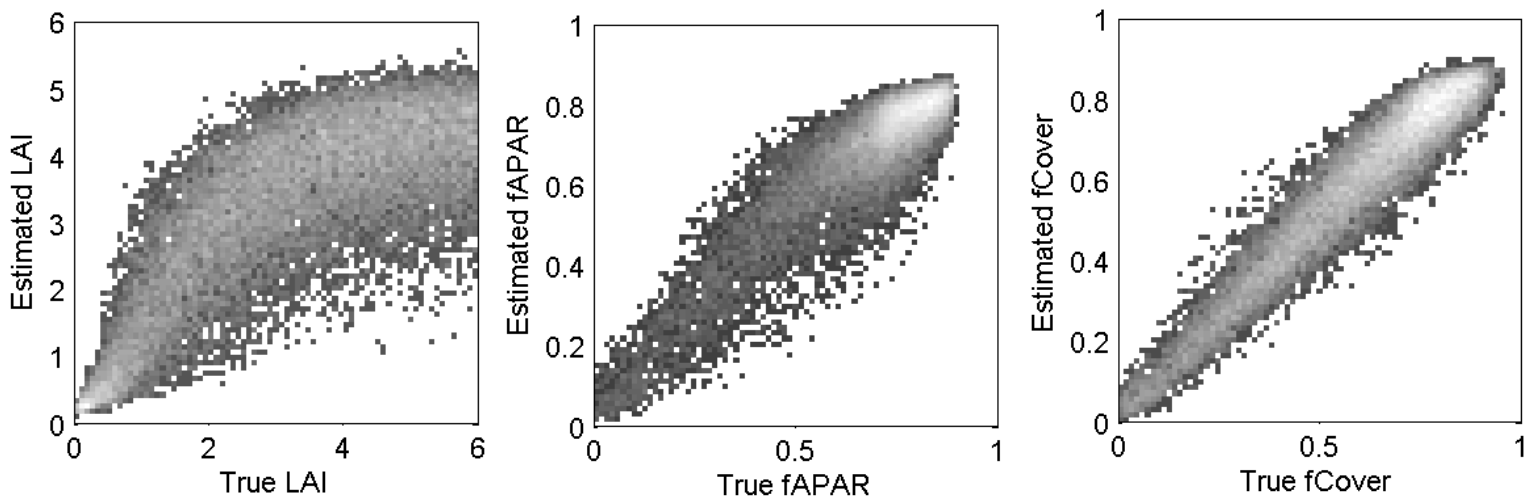

2 Figure 7. Density plots showing the theoretical performances evaluated over the validation data set.

4 A theoretical error model was adjusted based on the residuals computed over the validation data set (Figure 8). It shows an increase of the uncertainties up to $L A I$ values close to 3.0 , and then a stabilization showing however an underestimation of the actual $L A I$ values. This behaviour results from the non linearity of the LAI-reflectance relationship which saturates for the larger LAI values, i.e. reflectance is little sensitivite to LAI variation. In the mean time, some other variables such as canopy structure (ALA, hot) and leaf characteristics $\left(\mathrm{C}_{\mathrm{ab}}\right.$ in the red band, $\mathrm{C}_{\mathrm{w}}$ in the SWIR band, leaf mesophyll structure parameter, N) still impact reflectance values. The effect of these variables may be confounded with LAI effects, increasing the uncertainties attached to LAI retrievals. However, some regularization due to the distribution of LAI values in the training database tends to make the solution closer to the mode of LAI distribution, resulting in an underestimation of LAI for the larger LAI values.

For $f A P A R$ and fCover, uncertainties are relatively stable over the whole dynamic range.
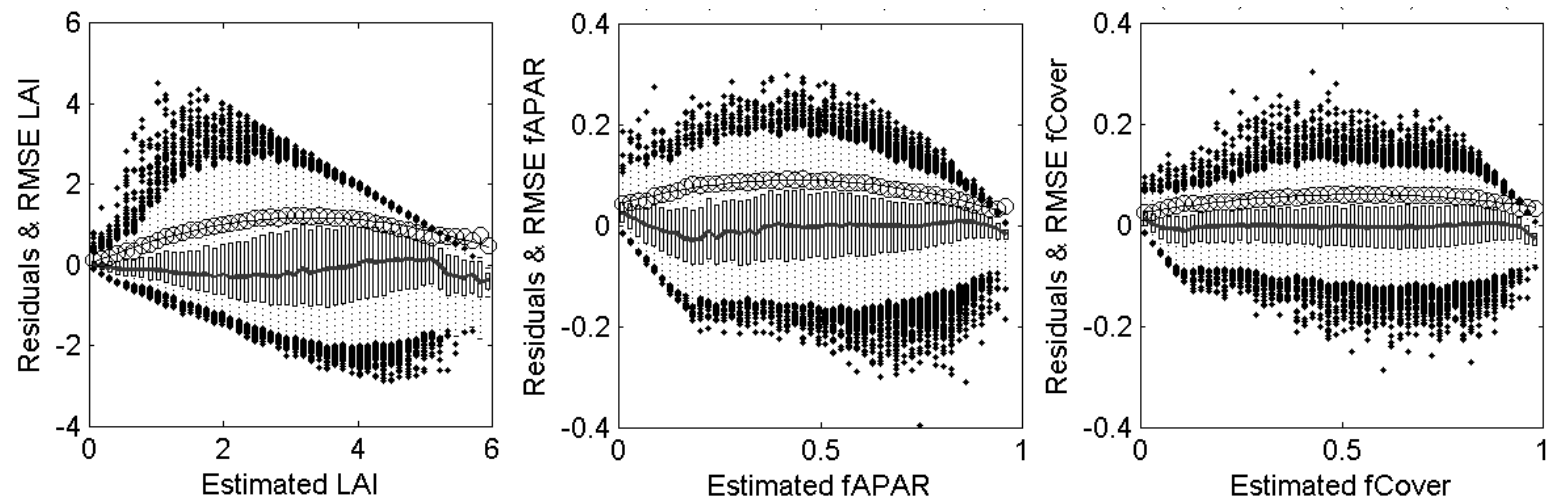

Figure 8. Distribution of the residuals (bars correspond to $25 \%$, dots to individual values below $1 \%$ or over 99\%. The solid line corresponds to the median value. The circles represent the RMSE values and thin solid line the corresponding uncertainty model.

\subsubsection{Management of extreme cases}

21 Minimum and maximum values in the training data base are used to declare 'out of range' the cases for which reflectance values in one band is outside this definition domain: 
Table 4 provides the values used to declare the inputs 'out of range'. Management of estimated values $(Y)$ falling outside the definition range of the considered biophysical variable was achieved similarly as previously, except that a tolerance value $\left(Y_{\text {tol }}\right)$ is used to keep slightly negative values by resetting them to $Y_{\min .}$ :

The use of the tolerance value was introduced to account for the uncertainties of the algorithm for the lower amount of vegetation possibly due to approximations in the description of soil reflectance properties. Table 4 provides the values of $Y_{\text {tol }}, Y_{\min }$ and $Y_{\max }$ for the three output biophysical products. Note that $L A I$, fAPAR and fCover are flagged as 'invalid' over snow and set to 0.00 over lakes.

\begin{tabular}{|l|c|c|c|}
\hline Inputs & $B 2$ & $B 3$ & $B 4$ \\
\hline$X_{\min }$ & 0.000 & 0.000 & 0.000 \\
\hline$X_{\max }$ & 0.612 & 0.916 & 0.835 \\
\hline & & & \\
\hline
\end{tabular}

\begin{tabular}{|c|c|c|c|}
\hline Outputs & $L A I$ & fAPAR & fCover \\
\hline$Y_{\min }$ & 0.00 & 0.00 & 0.00 \\
\hline$Y_{\max }$ & 6.00 & 1.00 & 1.00 \\
\hline$Y_{\text {tol }}$ & -0.20 & -0.10 & -0.10 \\
\hline
\end{tabular}

Table 4. On the left, $X_{\min }$ and $X_{\max }$ reflectance values used to declare the inputs 'out of range' in each of the three bands. On the right, $Y_{\min }, Y_{\max }$ and $Y_{\text {tol }}$ values used for LAI, fAPAR and fCover to declare the outputs 'out of range'.

\section{Conclusion}

CYCLOPES processing chains have been developed under a flexible and modular environment based on an open architecture (C++ code, CORBA integration, Java interfaces, PostgreSQL database backend). Using object-oriented components eases the integration of new algorithms while providing a reliable production framework. The actual processing is operated by 8 linux workstations (Intel 32 Architecture). The processing chains have been carefully implemented and allow producing about one year of global data within 27 days. The first processing steps, particularly those regarding cloud masking and atmospheric correction takes about 18 days, while BRDF normalization and compositing require about 8 days. The last step corresponding to the biophysical algorithm starting from the normalized reflectances to generate $L A I, f A P A R$ and fCover products requires only 1day processing per year of global data. This allows relatively fast reprocessing of this last step. This is achieved thanks to the efficiency of the neural network approach that offers great potentials for this type of application. 
1 CYCLOPES products derived from VEGETATION sensors are available freely at the following web

2 site: http://postel.mediasfrance.org/. The period 1998-2003 is currently available, and the series will soon be extended up to 2006 making 9 years of continuous and consistent observations. Products are organized by tiles of $10^{\circ}$ zenith and $10^{\circ}$ azimuth. One year of global data including the original $P$ products, intermediate products and the final LAI, fAPAR and fCover products representing $1.1 \mathrm{~Tb}$.

The current version 3 of CYCLOPES products have been validated by comparison to ground measurements and inter-comparison to other products. The corresponding results are presented in a companion paper (Weiss et al., 2007). Further, complementary validation activities were conducted (Garrigues et al., 2007), showing the inter-comparison with additional products as well as investigating the spatial consistency of the products.

The first steps of the processing seem relatively mature although some tuning may still improve the performances. Cloud masking could be improved by enhanced adjustment of the threshold values for each surface type. Additionally, distinction between snow, bare surfaces, water bodies and clouds may be also useful for the biophysical algorithms. Atmospheric correction could be improved by getting a better climatology of aerosol optical thickness and types. However, departure from the climatology due to events such as fires or sand storms, could only be corrected by developing autonomous correction methods providing that enough information is carried in the radiometric signal. The compositing and BRDF normalization method could also be improved with emphasis on gap filling to get continuous fields of variables as desired by most users. Kalman filtering as proposed by (Samain et al., 2007) may offer a very attractive solution. Nevertheless, largest improvements may come from the biophysical algorithm itself. The turbid medium canopy radiative transfer model does not describe leaf clumping, except at the landscape level through the vCover variable. Alternative models accounting for clumping at the tree and shoot levels could probably improve the results and allow retrieving higher values of LAI. However, it is not yet clear whether the measured radiometric signal carries enough and specific information on these additional canopy structure features. A prior classification may be used to get the required information to account for leaf clumping. Major enhancement of the algorithm is expected to come from a better design of the training data base. This should incorporate more realistic description of measurements and model uncertainties, particularly regarding the structure of these uncertainties, as well as distribution of the variables more representative of the actual ones. Further work is currently conducted in this direction, combining actual satellite observations and radiative transfer models to generate a better training data base.

\section{Acknowledgements:}


1 The CYCLOPES project was funded by the European Commission under the $5^{\text {th }}$ framework 2 programme under contract EVG2-2001-00035. Complementary funding was also provided by the 3 French Ministry of research under 'réseau terre-espace' programme. We thank VITO for providing the 4 VEGETATION data, CNES and POSTEL for the implementation and processing of the products. 5 Many thanks also to Kathy Pavageau and Cédric Bacour who contributed to the development of these 6 products. We thank AERONET PIs and staff for providing calibrated and processed data to this study, 7 and Météo-France for the synoptic cloudiness database provision. 


\section{References}

Abuelgasim, A. A., S. Gopal, and A. H. Strahler. 1998. Forward and inverse modelling of canopy directional reflectance using a neural network. International Journal of Remote Sensing, 19:453-471.

Atkinson, P. M., and A. R. L. Tatnall. 1997. Neural network in remote sensing. International Journal of Remote Sensing, 18:699-709.

Bacour, C., F. Baret, D. Béal, M. Weiss, and K. Pavageau. 2006. Neural network estimation of LAI, fAPAR, fCover and LAIxCab, from top of canopy MERIS reflectance data: principles and validation. Remote Sensing of Environment, 105:313-325.

Baret, F., and S. Buis. 2007. Estimating canopy characteristics from remote sensing observations. Review of methods and associated problems. In S. Liang (ed.), Advances in Land Remote Sensing: System, Modeling, Inversion and Application. Springer. (accepted)

Baret, F., J. G. P. W. Clevers, and M. D. Steven. 1995. The robustness of canopy gap fraction estimates from red and near infrared reflectances: a comparison of approaches. Remote sensing of the environment, 54:141-151.

Baret, F., M. Leroy, O. Hagolle, P. Bicheron, J. L. Roujean, M. Weiss, C. Bacour, B. Geiger, B. Berthelot, R. Lacaze, O. Samain, F. Nino, B. Miras, D. Béal, and G. Derive. 2004. Development of high level biophysical products from the fusion of medium resolution sensors for regional to global applications: the CYCLOPES project, ENVISAT workshop, Salzburg (Austria).

Baret, F., J. Morissette, R. Fernandes, J. L. Champeaux, R. Myneni, J. Chen, S. Plummer, M. Weiss, C. Bacour, S. Garrigue, and J. Nickeson. 2006. Evaluation of the representativeness of networks of sites for the global validation and inter-comparison of land biophysical products. Proposition of the CEOS-BELMANIP. IEEE Transactions on Geoscience and Remote Sensing, 44:1794-1803.

Bartholomé, E., and A. S. Belward. 2005. GLC2000: a new approach to global land cover mapping from Earth Observation data. International Journal of Remote Sensing, 26: 1959 - 1977.

Béal, D., F. Baret, C. Bacour, and X. F. Gu. 2006. Development and validation of an algorithm for aerosol optical depth estimates from MERIS Level1B images. International Journal of Remote Sensing, in press. 
1 Berthelot, B. 2004. Snow detection on VEGETATION data. Improvement of cloud screening, pp. 54. Noveltis, Toulouse.

Berthelot, B., and G. Dedieu. 1997. Correction of atmospheric effects for vegetation data. In G. Guyot and T. Phulpin (eds.), Physical measurements and signatures in Remote Sensing, pp. 19-25. Balkema, Courchevel (France).

Berthelot, B., A. Quesney, O. Hagolle, F. Cabot, and V. Bruniquel. 2004. The CYCLOPES project: cloud screening and atmospheric correction, VEGETATION users conference, Antwerp (Belgium).

Cabot, F., O. Hagolle, and P. Henry. 2000. Relative and multitemporal calibration of AVHRR, SeaWiFS, and VEGETATION using POLDER characterization of desert sites., IGARSS 2000, Vol. 5, pp. 2188 - 2190, Hawaii (USA).

Campbell, G. S. 1990. Derivation of an angle density function for canopies with ellipsoidal leaf angle distribution. Agricultural and Forest Meteorology, 49:173-176.

Cosnefroy, H., X. Briottet, and M. Leroy. 1996. Selection and characterization of Sahara and Arabian desert sites for the calibration of optical satellite sensors. Remote Sensing of Environment, 58:101-114.

Danson, F. M., C. S. Rowland, and F. Baret. 2003. Training a neural network with a canopy reflectance model to estimate crop leaf area index. International Journal of Remote Sensing, 24:4891-4905.

Dozier, J. 1989. Spectral signature of alpine snow cover from Landsat 5 TM. Remote Sensing of Environment, 28:9 - 22.

Fang, H., and S. Liang. 2005. A hybrid inversion method for mapping leaf area index from MODIS data: experiments and application to broadleaf and needleleaf canopies. Remote Sensing of Enviroment, 94:405-424.

Fourty, T., and F. Baret. 1997. Amélioration de la précision des coefficients d'absorption spécifique de la matière sèche et des pigments photosynthétiques, pp. 35. INRA Bioclimatologie, Avignon.

Garrigues, S., R. Lacaze, J. Morisette, F. Baret, M. Weiss, R. Fernandes, J. Nickeson, S. Plummer, W. Yang, and R. Myneni. 2007. The CEOS Leaf Area Index Intercomparison as a prototype activity. Remote Sensing of Enviroment, in preparation.

Geiger, B., O. Hagolle, and P. Bicheron. 2006. Algorithm Theoretical Basis Document: Directional Normalisation. Version 3.0. CYCLOPES Project. CNRM, Toulouse. Gong, P., D. X. Wang, and S. Liang. 1999. Inverting a canopy reflectance model using a neural network. International Journal of Remote Sensing, 20:111-122. 
Hagolle, O., P. Goloub, P.-Y. Deschamps, H. Cosnefroy, X. Briottet, T. Bailleul, J.-M. Nicolas, F. Parol, B. Lafrance, and M. Herman. 1999. Results of POLDER in-flight calibration. IEEE Transactions on Geoscience and Remote Sensing, 37:1550-1566.

Hagolle, O., A. Lobo, P. Maisongrande, F. Cabot, B. Duchemin, and A. De Pereyra. 2005. Quality assessment and improvement of temporally composited products of remotely sensed imagery by combination of VEGETATION 1 and 2 images. Remote Sensing of Environment, 94:172-186.

Hagolle, O., J.-M. Nicolas, B. Fougnie, F. Cabot, and P. Henry. 2004. Absolute calibration of VEGETATION derived from an interband method based on the Sun glint over ocean. IEEE Transactions on Geoscience and Remote Sensing, 42:1472 -1481.

Harrel, F. E. 2001. Regression modeling strategies. Springer, New-York.

Henry, P. 1999. The VEGETATION system: a global monitoring system onboard SPOT4. In ESA (ed.), Euro-Asia space week on cooperation in space, Vol. ESA-SP 430, pp. 233239.

Holben, B. N., D. Tanre, A. Smirnov, T. F. Eck, I. Slutsker, N. Abuhassan, W. W. Newcomb, J. Schafer, B. Chatenet, F. Lavenu, Y. J. Kaufman, J. Vande Castle, A. Setzer, B. Markham, D. Clark, R. Frouin, R. Halthore, A. Karnieli, N. T. O'Neill, C. Pietras, R. T. Pinker, K. Voss, and G. Zibordi. 2001. An emerging ground-based aerosol climatology: Aerosol Optical Depth from AERONET. Journal of Geophysical Research, 106:12067-12097.

Jacquemoud, S., and F. Baret. 1990. PROSPECT: A model of leaf optical properties spectra. Remote Sensing of Environment, 34:75-91.

Kaufman, Y. J., D. Tanré, L. A. Remer, E. F. Vermote, A. Chu, and B. N. Holben. 1997. Operational remote sensing of tropospheric aerosol over land from EOS moderate resolution imaging spectroradiometer. Journal of Geophysical Research, 102:1705117067.

Kempeneers, P., G. Lissens, and F. Fierens. 2000. Development of a cloud, snow and cloud shadow mask for VEGETATION imagery. In G. Saint (ed.), Vegetation 2000, 2 years of operation to prepare the future, pp. 303-306, Belgirate, Italy.

Kimes, D. S., R. F. Nelson, M. T. Manry, and A. K. Fung. 1998. Attributes of neural networks for extracting continuous vegetation variables from optical and radar measurements. International Journal of Remote Sensing, 19:2639-2663.

Kuusk, A. 1991. The hot-spot effect in plant canopy reflectance, Tartu University. 
Lacaze, R. 2005. POLDER-2 Land surface level 3 products. User manual and product validation, pp. 70. Medias/CNES, Toulouse.

Leshno, M., V. Ya Lin, A. Pinkus, and S. Shocken. 1993. Multilayer feedforward networks with non polynomial activation function can approximate any function. Neural Networks, 6:861-867.

Levy, R. C., L. A. Remer, J. V. Martins, Y. J. Kaufman, A. Plana-Fattori, J. Redeman, and B. Wenny. 2005. Evaluation of the MODIS aerosol retrievals over ocean and land during CLAMS. Journal of the Atmospheric Sciences, 62:974-992.

Liu, W., F. Baret, X. F. Gu, B. Zhang, Q. Tong, and L. Zhang. 2003. Evaluation of methods

Maisongrande, P., B. Duchemin, and G. Dedieu. 2004. VEGETATION/SPOT: an operational 13 for soil surface moisture estimation from reflectance data. International Journal of Remote Sensing, 24:2069-2083. mission for the Earth monitoring; presentation of new standard products. International Journal of Remote Sensing, 25:9 - 14.

Myneni, R. B., S. Hoffman, Y. Knyazikhin, J. L. Privette, J. Glassy, Y. Tian, Y. Wang, X. Song, Y. Zhang, G. R. Smith, A. Lotsch, M. Friedl, J. T. Morisette, P. Votava, R. R. Nemani, and S. W. Running. 2002. Global products of vegetation leaf area and absorbed PAR from year one of MODIS data. Remote Sensing of Environment, 83:214-231.

Ngia, L. S. H., and J. Sjoberg. 2000. Efficient training of neural nets for nonlinear adaptive filtering using a recursive Levenberg-Marquardt algorithm. IEEE Transactions on Signal Processing, 48:1915-1927.

Plummer, S., O. Arino, F. Fierens, G. Borstlap, G. Dedieu, F. Ranera, and M. Simon. 2005. The GLOBCARBON initiative: multi-sensor estimation of global biophysical products for global terrestrial carbon studies, MERIS (A)ATSR workshop 2005, Frascati (Italy).

Rahman, H., and G. Dedieu. 1994. SMAC: a simplified method for the atmospheric correction of satellite measurements in the solar spectrum. International Journal of Remote Sensing, 15:123-143.

Roujean, J. L., M. Leroy, and P. Y. Deschamps. 1992. A bidirectional reflectance model of the Earth's surface for the correction

of remote sensing data. Journal of geophysical research, 97:20455-20468. 
1 Rummelhart, D. E., G. E. Hinton, and R. J. Williams. 1986. Learning internal representations by error propagation. In D. Rummelhart and J. Mc Clelland (eds.), Parallel data processing, Vol. 1, pp. 318-362. M.I.T. press, Cambridge, MA (USA).

Samain, O., J. L. Roujean, and B. Geiger. 2007. Use of Kalman filter for the retrieval of surface BRDF coefficients with time-evolving model based on ECOCLIMAP land cover classification. Remote Sensing of Environment, submitted.

Smith, J. A. 1992. LAI inversion from optical reflectance using neural network trained with multiple scattering model. In W. R. (ed.), IGARS'92, pp. 757-759, South Shore Harbour Resort and Conference center, Houston, texas, USA.

—. 1993. LAI inversion using backpropagation neural network trained with multiple scattering model. IEEE Transactions on Geoscience and Remote Sensing, 31:11021106.

Sylvander, S., I. Albert-Grousset, and P. Henry. 2003. Geometrical performances of the VEGETATION products, IGARSS 2003, Vol. CD. IEEE, Toulouse (France).

Verhoef, W. 1984. Light scattering by leaf layers with application to canopy reflectance modeling: the SAIL model. Remote Sensing of Environment, 16:125-141.

—. 1985. Earth observation modeling based on layer scattering matrices. Remote Sensing of Environment, 17:165-178.

Weiss, M., F. Baret, S. Garrigues, R. Lacaze, and P. Bicheron. 2007. LAI, fAPAR and fCover CYCLOPES global products derived from VEGETATION. part 2: Validation and comparison with MODIS Collection 4 products. Remote sensing of Environment, submitted.

Weiss, M., F. Baret, M. Leroy, O. Hautecoeur, C. Bacour, L. Prévot, and N. Bruguier. 2002. Validation of neural net techniques to estimate canopy biophysical variables from remote sensing data. Agronomie, 22:547-554. 